\title{
A neurosurgical navigation system based on intraoperative tumour remnant estimation
}

\author{
Jaesung Hong · Yoshihiro Muragaki • \\ Ryoichi Nakamura · Makoto Hashizume • \\ Hiroshi Iseki
}

Received: 17 October 2006/ Accepted: 10 January 2007 / Published online: 10 February 2007

(C) Springer London 2007

\begin{abstract}
This paper proposes a method to intraoperatively visualize the process of tumour resection until complete resection is accomplished. A fuzzy connectedness method that is robust against image noises was used to identify the tumour position and volume. Based on the tumour segmentation results, the removed area and the residual tumour tissues were examined with reference to the electrocautery trace $\log$. Unique processes that are specific to glioma resection were introduced in the method to improve the accuracy of estimation. Invalid data in the trace log were excluded, and the tumour region surrounded by valid $\log$ points was included in the removed area. The proposed system also produces an alarm to indicate whether the electrocautery is being accurately performed within the tumour area. Thus, this surgical navigation system can assist surgeons in intuitively monitoring the tumour resection process and properly removing tumour remnants. Preliminary experiments and a clinical pilot study showed the feasible application of this method.
\end{abstract}

\footnotetext{
J. Hong $(\square)$

Department of Nanobiomedicine,

Faculty of Medical Sciences, Kyushu University,

Fukuoka, Japan

e-mail: hong@dem.med.kyushu-u.ac.jp

Y. Muragaki · R. Nakamura · H. Iseki

Institute of Advanced Biomedical Engineering and Science,

Tokyo Women's Medical University, Tokyo, Japan

M. Hashizume

Department of Advanced Medicine and Innovative

Technology, Kyushu University Hospital,

Fukuoka, Japan
}

Keywords Surgical navigation · Image-guided surgery - Display of residual tumour - Trace analysis of electrocautery · Tumour segmentation

\section{Introduction}

In neurosurgery, the tumour resection rate significantly affects patient survival. According to the Japan tumour registry 2000 , the data of survival following malignant glioma revealed that only complete resection could improve the five-year survival rate (up to $40 \%$ ). With regard to the survival rate, the cases of $95-99 \%$ resection were not considerably different from those in which no resection was performed. To aid $100 \%$ tumour removal without invasion of the adjacent normal tissues, computer-aided procedures such as surgical navigation have been introduced $[1,2]$.

Further, intra-operative magnetic resonance imaging (MRI) has been widely used in the field of neurosurgery since the late 1990s. It provides the surgeon with updated images of the lesion during surgery, thus facilitating the verification of the treatment process in progress. Therefore, the combined use of a surgical navigation system and intra-operative MRI will allow surgeons to accurately approach the target area that is mapped on the updated MR images.

However, a number of navigation systems using intra-operative MRI mainly provide the surgeon with a series of two-dimensional (2D) images [3-6]. Surgeons are frequently required to observe the navigation monitor while approaching the tumour margins, and they must imagine three-dimensional (3D) objects based on 2D images. Although several previous navigation systems and relative registration techniques 
appeared to provide 3D volume-rendering options, they could not identify specific areas such as tumour remnants, which surgeons require to discriminate between the tumour and the surrounding normal tissues [7-9]. Another study focused on a pre-operative diagnosis, although it provided segmentation and a 3D image of the region of interest (ROI) [10]. To our knowledge, the intra-operative computation and visualization of tumour remnants on the basis of the trace $\log$ of electrocautery have not been reported thus far.

To improve previous navigation systems, we added a tumour segmentation module and an analyzer to the electrocautery trace $\log$. Since the trace analyzer provides details on the removed volume and residual tumour tissues without additional MR scanning, the surgeon can reduce the number of scans, which would otherwise extend the total surgery time.

From the navigation display, the surgeons can intuitively identify the removed and residual tumour areas presented as 3D coloured models. This information can aid surgeons in complete removing tumour remnants. By producing a specific sound, our proposed system can also inform surgeons about whether they are working within the tumour area without disturbing the adjacent normal tissues; thus, surgeons can concentrate on the resection procedure without shifting their focus from the treatment area.

\section{Materials and methods}

In the present study, we applied our method to the data of five patients. In the Tokyo Women's Medical University Hospital, these patients underwent MRI-guided surgery with the 0.3-Tesla intra-operative MR imager AIRIS II (HITACHI, Tokyo, Japan). Prior to the surgery, informed consent for the use of the surgical navigation system was obtained from all patients, and any personal information was considered completely confidential. The patient data is summarised in Table 1.

Three-dimensional MR images were obtained using the following parameters: TR 1,000 ms; TE $140 \mathrm{~ms}$;
NEX 1; matrix $256 \times 256$; and scan order, axial IS. The patients had to be moved into the MR scanner during scanning, and moved out for the treatment. During surgery, the normal number of scans is fewer than three or four. Therefore, most of the tumour should be removed before the second or third scan.

Figure 1 depicts the overall process incorporating the proposed method.

\section{Intra-operative segmentation of brain tumours}

Intra-operative segmentation and 3D visualization of brain tumours assist surgeons in intuitively identifying the tumour position and margins. They also facilitate the quantitative measurement of the tumour size and volume during surgery. We used the fuzzy connectedness (FC) method to perform intra-operative segmentation of brain tumours. The FC method was first proposed for application in medical image segmentation, followed by reports on tumour segmentation using MRI [11-12]. Starting from a selected seed point in the tissue of interest (i.e. tumour), the method calculates the affinity of the neighbouring voxels based on two voxel criteria: their spatial closeness and the similarity of their image intensity. The algorithm automatically computes a fuzzy scene (a map of fuzzy connectivity) from the grey-scale images.

The FC method employed in this study requires two parameters: a seed point and a threshold for the fuzzy scene. The operator sets the seed point in the region of the tumour by clicking on one pixel displayed on the monitor. The operator also limits the region of analysis by enclosing the entire tumour in a rectangle and inputting the numbers of the first and last slices. This procedure reduces the processing time. A threshold is then set in the precomputed fuzzy scene. The robustness of the FC method can overcome the noise problems that are inherent to MR images. Since the ROI can be determined using a graphical user interface (GUI), the required processing time is less than $10 \mathrm{~s}$ with a standard Linux PC (CPU: Pentium 4, $2.53 \mathrm{GHz}$; RAM: $1024 \mathrm{MB}$ ); this speed is acceptable for intraoperative use.

Table 1 Patient data

\begin{tabular}{llllll}
\hline Case & Age & Sex & Histological diagnosis & WHO grade & Location of the lesion \\
\hline A & 43 & F & Anaplastic oligoastrocytoma & 3 & rt frontal lobe \\
B & 39 & F & Astrocytoma & 2 & lt insula \\
C & 44 & F & Anaplastic astrocytoma & 3 & rt insula-frontal lobe \\
D & 30 & F & Astrocytoma & 2 & lt frontal \\
E & 52 & F & Glioblastoma & 4 & rt parietal lobe \\
\hline
\end{tabular}

$F$ female, $r t$ right, $l t$ left 


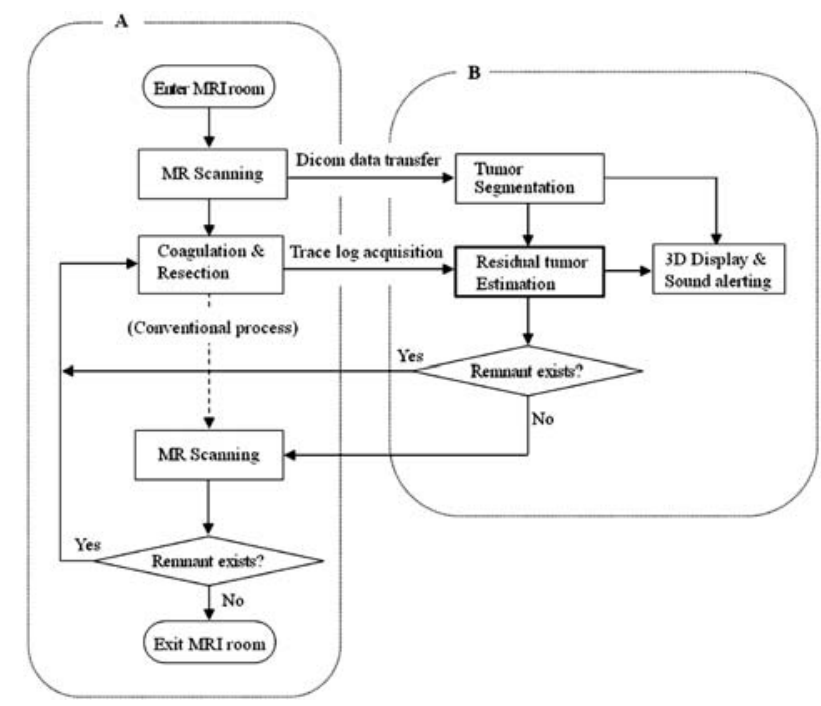

Fig. $1 A$ represents a conventional process; $B$ represents the proposed process incorporated

To evaluate the performance of the FC segmentation, manual segmentation was performed by an expert neurosurgeon, and manual segmentation results were assumed to the standard. The neurosurgeon outlined the tumour boundaries on the images, and these were used for the comparison with the automatic segmentation. From the manual and automatic segmentation results, we calculated the percent match (PM) and dice similarity coefficient (DSC), both of which are equal to 1.0 when the results are perfectly matched [13].

\section{Calculation and display of tumour remnants}

The trace log can be obtained from the optical sensor Polaris (NDI, ON, Canada) that recognizes the position of the surgical device, to which optical markers are attached. Surgical procedures for brain tumour resection usually involve coagulation followed by cutting; therefore, the area that is coagulated using electrocautery can be regarded as the removed area. The trace recording in our system was updated at $1.5 \mathrm{~Hz}$. The current method is applicable only when electrocautery is used for the resection process. For cases that do not require electrocautery, optical markers need to be attached to the different forceps that surgeons use, to collect the necessary trace data.

To estimate the removed area from the limited record of the trace log accurately, several additional processes were attempted as follows:

Setting of coagulation efficiency Since the trace of the $\log$ data consisted of a number of points with coordinates corresponding to the electrocautery position, the coagulated volume can be calculated from these points. The effects of coagulation on the tissues differs depending on the type and specifications of electrocautery. A parameter was designed for the setting of coagulation efficiency, and an appropriate value is chosen for each case.

Exclusion of invalid trace log Irrelevant data from the trace of log points should be eliminated. The trace $\log$ that is obtained while the surgeon rapidly moves the electrocautery device from one point to another should be excluded because the coagulation that occurs during this motion is insufficient or absent. This process involves checking the movement speed of the electrocautery device. This speed can be straightforwardly measured from the recorded positions in the trace $\log$ data. Finally, we select points that are located within the tumour and eliminate points that lie outside the tumour.

Inclusion of surrounded area To calculate the removed volume from the valid trace log, we constructed a volume by connecting each voxel that is regarded as being coagulated. However, this process yields an incomplete volume that does not include the interior of the tumour because surgeons tend to remove the brain tumour by accessing the tumour boundary without complete coagulation of the interior of the tumour, i.e., the interior of the tumour is actually removed without any trace log being recorded. Therefore, we added another process to include the interior of the tumour in the removed area. First, the centroid of the tumour is calculated from the segmentation results as described previously; then, each voxel adjacent to the line that connects the centroid to each valid log point is regarded as coagulated. By this process, we included the interior of the tumour where no trace log was recorded (Fig. 2).

Once the removed area is calculated, the residual tumour tissues are simply estimated by subtracting the removed area from the tumour segmentation results.

Finally, the removed and residual tumour areas are displayed in 3D and made available to the surgeons (Fig. 3). The open-source navigation software 3D Slicer (Brigham \& Women's Hospital, Boston, USA) was employed to verify the clinical feasibility of the proposed method.

To validate the process of estimation and display of tumour remnants, we performed four retrospective studies and one clinical application.

Guidance for proper access to residual tumour tissues

By segmenting the tumour and estimating the remnant tumour area, surgeons can intuitively decide whether 
Fig. 2 The interior of the tumour where no trace log was recorded is included in the removed area. The red points represent the valid log points, the pink points represent the coagulation area, and the green area represents the removed area that was calculated from the trace $\log$
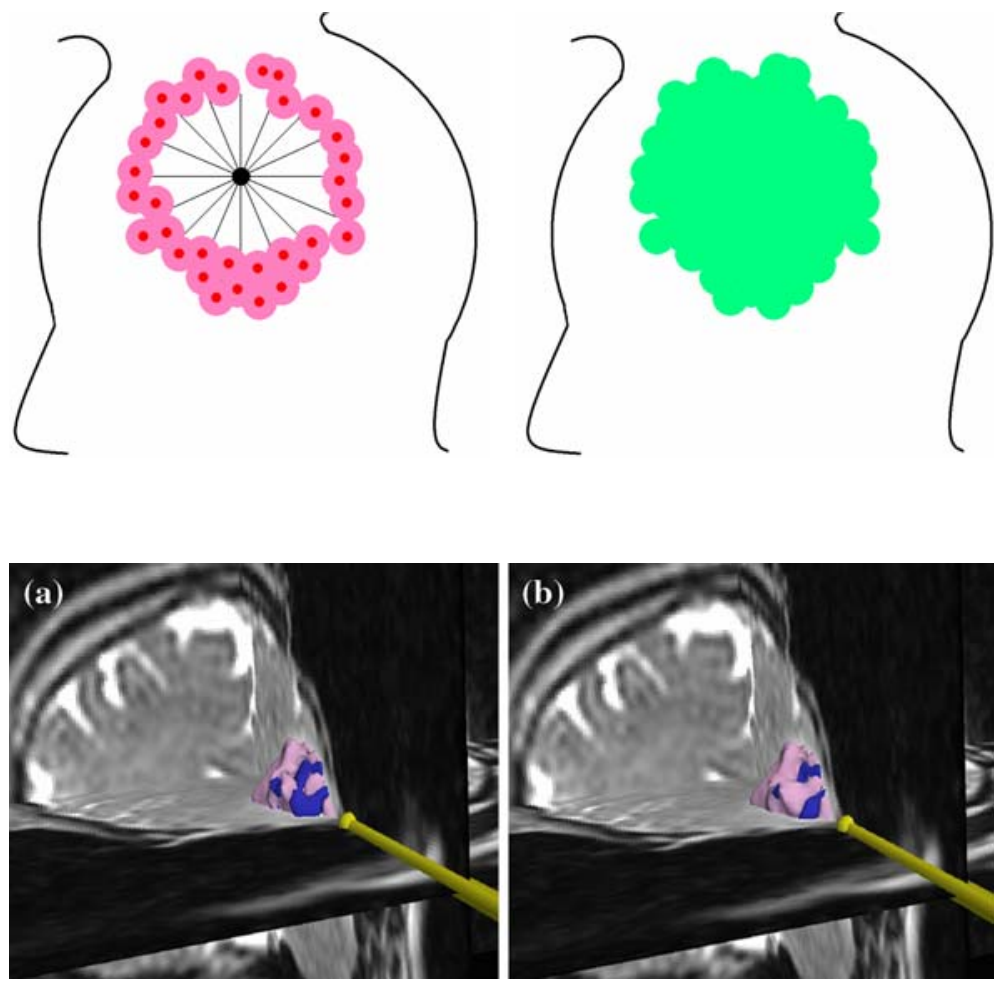

Fig. 3 Three-dimensional display of tumour resection. a The tumour in the early stage of the operation. The blue area represents the residual tumour tissue, and the pink area represents the coagulated and removed tissue. b The tumour in the late stage of the operation. Note that the residual tumour area is reduced in (b) additional treatment is necessary, as shown in Fig. 3. However, when surgeons follow the navigation display, they cannot directly observe the tumour in the patient, and vice versa. This problem could disrupt the surgeons' concentration during surgery.

We developed a system that produces an alarm when the electrocautery device is located within the tumour. Thus, the surgeons could continue operating without shifting their focus from the patient. They can easily know whether they are working within or outside the tumour based on the alarm.

Since the resection time was short in the total surgical time, the surgeons set the system such that it sounded an alarm when the electrocautery device was inside the tumour rather than outside it.

\section{Results}

From the segmentation results, the average PM and DSC were 0.82 and 0.83 , respectively. Tumour volume was also automatically calculated based on these results. The surgeon could track the size of the brain tumour and estimate the extra operation time on the basis of the tumour size. Figure 4 shows one of the segmentation results.

Figure 5 shows the effects of two additional processes that were proposed in this study for the accurate estimation of tumour remnants. The result presented in
Fig. 5 was obtained from the data of clinical case $\mathrm{E}$ in which the trace log was collected carefully. Although the excluded invalid log data appeared to underestimate the removed area, it was considered to reflect the actually removed area but not the area that was merely in contact with the electrocautery device. For data from five patients, assuming that the resection rate by no additional process was 1.0 , the resection rate by two additional processes was $1.16 \pm 0.18$. These processes were found to improve the accuracy of the estimation by the surgeons.

Figure 6 presents the changes in the rate at which the tumour tissue remained between the first and second scans during surgery. We observed the residual tumour tissues assumed to exist when the surgeons ended a resection following the first scan.

The sound alert system was also applied in over 20 clinical cases; in a qualitative evaluation, surgeons concluded that the system was very useful during the removal of tumours.

\section{Discussion}

This study showed that brain tumour segmentation and the intra-operative display of residual tumour tissues could assist the surgeon in accomplishing accurate tumour resection. In addition, the sound alert system proved to be useful in clinical application. We also 
Fig. 4 Intra-operative brain tumour segmentation. a Initial setting for ROI. b Segmentation result; the red area represents the automatically segmented tumour, and the line represents the tumour boundary that was manually outlined by the surgeon

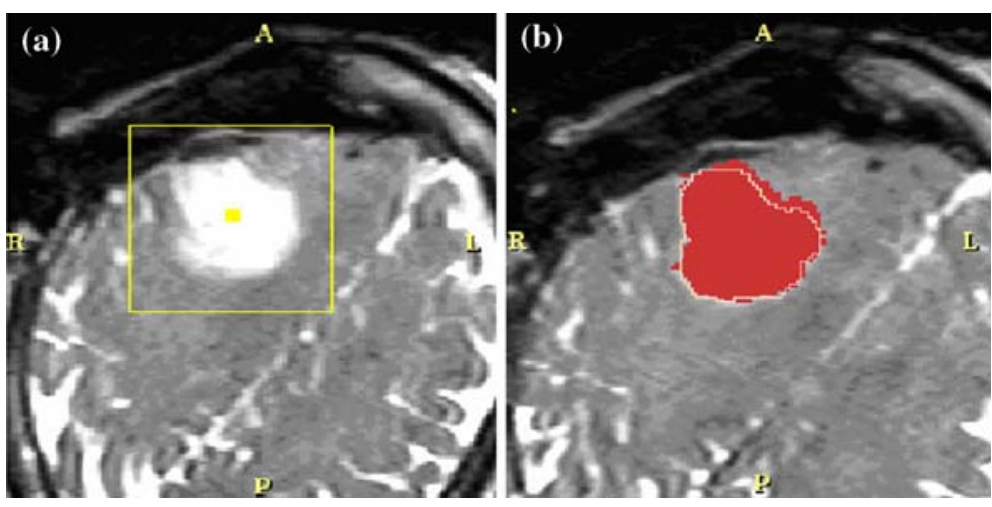

- - - - w: no additional process

- - O - - y:including surrounded area $-0-\mathrm{z}$ : both $\mathrm{x} \& \mathrm{y}$ applied

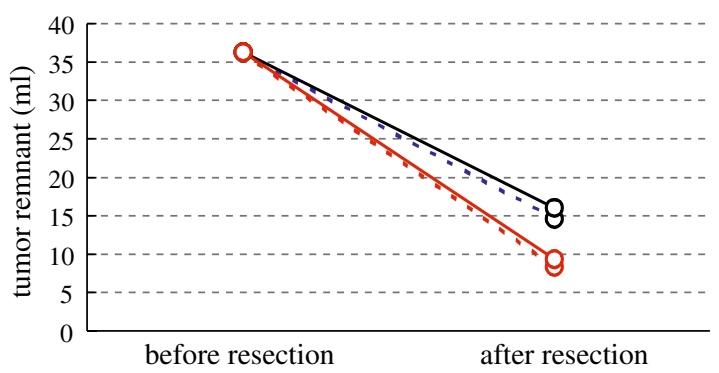

Fig. 5 Changes in tumour volume before the second scan. The excluding invalid trace $\log$ and the including the surrounded area by the valid trace $\log$ were applied for a more accurate estimation of the residual tumour tissues

- excluding invalid trace

-

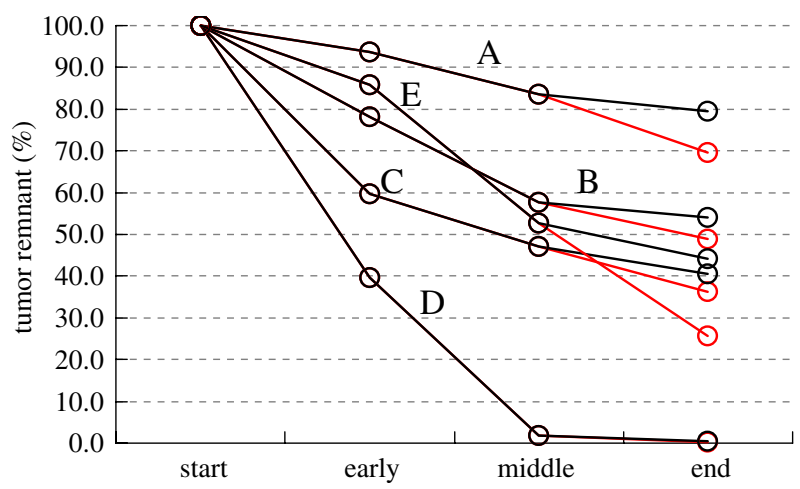

Fig. 6 Changes in the tumour tissue remaining during surgery in the five patients. After the surgeons conducted a resection following the first scan, the residual tumour tissue was estimated and displayed by analysing the trace log of electrocautery

proposed a useful process to estimate the tumour remnants accurately by excluding the invalid points in the trace $\log$ and including the surrounded area by the valid points. Although the final estimation still indicated considerable tumour remnant, for example over $25 \%$ in the case of $\mathrm{E}$, this result can provide information on the direction in which surgeons should approach after subsequent scans.

However, we observed that the segmentation module did not produce accurate results when the tumour margins were obscured by the presence of edemas, ventricles, etc. If these errors are unacceptable to the surgeons, before estimating the tumour remnants, the errors can be partly edited using a manual procedure. To improve the accuracy of this method, future studies will be required to include knowledge-based parameters in the FC calculation.

As an offshoot of tumour segmentation, if the segmentation results are successfully obtained, the segmented region in the T2-weighted image could be overlaid on that in the T1-weighted image. In T2weighted images, brain tumours show high intensities while other tissues are unclear; however, this is the opposite in the case of T1-weighted images. Therefore, overlaying the segmentation result in one image onto that of the other image can be advantageous.

Brain shift during surgery is another problem with regard to image-guided therapy [14-16]. Intra-operative MR scanning, however, provides the latest information following brain shift, which surgeons can observe at any time if required. Further, ultrasound could be an alternative modality for cases that require real-time observation of brain shift.

Figure 6 shows that, in several cases, surgeons could not remove approximately $50 \%$ of the tumour tissue before the second scan. This might represent an erroneous estimation even though following coagulations were assumed to be done with additional MR scanning. This is because the number of logs obtained from the optical sensor was too few for the system to estimate the removed and residual tumour tissues. Discontinuities in the trace $\log$ were due to the occurrence of obstacles in the visual path of the optical sensor, which 
Table 2 Coverage of trace logs in the five patients

\begin{tabular}{llllll}
\hline & A & B & C & D & E \\
\hline Tumour (ml) & 38.88 & 33.57 & 36.00 & 11.74 & 36.29 \\
No. of points & 3109 & 4271 & 6844 & 7456 & 15819 \\
Coverage & 79.97 & 127.22 & 184.99 & 634.93 & 435.91 \\
\hline
\end{tabular}

Data were collected from four retrospective studies (A-D) and one clinical application (E)

is termed a line-of-sight problem. For an accurate estimation, we must obtain a sufficient number of trace logs.

Table 2 presents the coverage rate, which was calculated by dividing the tumour volume by the number of points from the trace log data as defined by,

Coverage $=\frac{\text { tumour volume }}{\text { number of trace points }}$

The data used for the retrospective studies had a small number of recorded points because the trace log data were not carefully collected before the proposed system was introduced. Therefore, the tumour remnant estimation was incomplete. In the clinical case, however, the data were carefully collected, and this case showed better estimation results.

To estimate the residual tumour tissues accurately, we considered a coverage of over 400 as necessary, based on the experiments. If the coverage is less than 400 , the additional collection of trace data would be required. Therefore, during the surgery, sufficient trace log data should be carefully collected taking into consideration the visual path of the optical sensor. To overcome the line-of-sight problem, a magnetic position sensor can be used as an alternative tool, provided no magnetic disturbance occurs in the MRI room.

In this study, the percentages of tumor resection were defined by tumor identification based on MR images. Even if $100 \%$ resection is accomplished, glioma cells infiltrated within normal brain could still exist. However, $98 \%$ or more resection has been reported with significant survival advantage [17].

\section{Conclusion}

This study proposed a method for the intra-operative 3D display of the status of brain tumour resection to achieve accurate tumour removal. The intra-operative segmentation process reveals information on the tumour position and volume. Based on the tumour segmentation results, the removed and residual tumour areas are estimated using the trace $\log$ data of electrocautery. Therefore, surgeons can accurately approach the residual tumour tissues before or without additional MR scanning.

The production of an alarm when the tumour area is accessed was also useful. The results of four retrospective and a clinical pilot studies indicated the clinical feasibility of this method.

Acknowledgments The segmentation module was mainly developed by Mr. Takashi Inomata who graduated from the Graduate School of Information Science and Technology, University of Tokyo in 2005 . We greatly appreciate his contribution.

\section{References}

1. Schulz T, Puccini S, Schneider J-P, Kahn T (2004) Interventional and intraoperative MR: review and update of techniques and clinical experience. Eur Radiol 14:2212-2227

2. Vannier MW, Haller JW (1999) Navigation in diagnosis and therapy. Eur J Radiol 31:132-140

3. Roberson PL, Mclaughlin PW, Narayana V, Troyer S, Hixson GV, Kessler ML (2005) Use and uncertainties of mutual information for computed tomography/magnetic resonance $(\mathrm{CT} / \mathrm{MR})$ registration post permanent implant of the prostate. Med Phys 32(2):473-482

4. Muragaki Y, Amano K, Iseki H, Kawamata T, Maruyama T, Takakura K, Hori T (2001) Initial experience of intraoperative MR imaging and "real-time" navigation. Societe Francaise De Neurochirurgie 15

5. Muragaki Y, Iseki H, Hori T (2001) The cerebral preservation neurosurgery using the navigator and open MRI. Symposium-brain mapping for surgical strategy. International Society for Brain Electromagnetic Topography 12th World Congress 28

6. Hiroshi I (2000) Image guided brain surgery. In: The 3rd Asian conference of neurological surgeons L17-2

7. Bao P, Warmath J, Galloway R Jr, Herline A (2005 Ultrasound-to-computer-tomography registration for image-guided laparoscopic liver surgery. Surg Endosc 19:424-429

8. Pagoulatos N, Edwards WS, Haynor DR, Kim Y (1999) Interactive 3D registration of ultrasound and magnetic resonance images based on a magnetic position sensor. IEEE Trans Inf Technol biomed 3(4):278-288

9. Tronnier VM, Bonsanto MM, Staubert A, Knauth M, Kunze S, Wirtz CR (2001) Comparison of intraoperative MR imaging and 3D-navigated ultrasonography in the detection and resection control of lesions. Neurosurg Focus 10(2):1-4

10. Slomka PJ, Mandel J, Downey D, Fenster A (2001) Evaluation of voxel-based registration of 3D power Doppler ultrasound and 3D magnetic resonance angiographic images of carotid arteries. Ultrasound Med Biol 27(7):945-955

11. Udupa JK, Samarasekera S (1999) Fuzzy connectedness and object definition: theory, algorithms, and applications in image segmentation. Graph Models Img Proc 9:85-90

12. Moonis G, Liu J, Udupa JK, Hackney DB (2002) Estimation of tumor volume with fuzzy-connectedness segmentation of MR images. AJNR Am J Neuroradiol 23:356-363

13. Hata N, Muragaki Y, Inomata T, Maruyama T, Iseki H, Hori $\mathrm{T}$, Dohi $\mathrm{T}$ (2005) Intraoperative tumor segmentation and volume measurement in MRI-guided glioma surgery for tumor resection rate control. Acad Radiol 12(1):116-22 
14. Hejazi N (2005) Frameless image-guided neuronavigation in orbital surgery: practical applications. Neurosurg Rev 23:1-5

15. Reinges MH, Nguyen HH, Krings T, Hutter BO, Rohde V, Gilsbach JM (2004) Course of brain shift during microsurgical resection of supratentorial cerebral lesions: limits of conventional neuronavigation. Acta Neurochir (Wien) 146:369-377

16. Roberts DW, Hartov A, Kennedy FE, Miga M, Paulsen KD (1998) Intraoperative brain shift and deformation: a quanti- tative analysis of cortical displacement in 28 cases. Neurosurgery 43(4):749-758

17. Lacroix M, Fourney DR, Gokaslan ZL, Shi W, DeMonte F, Lang FF, McCutcheon IE, Hassenbusch SJ, Holland E, Hess K, Michael C, Miller D, Sawaya R (2001) A multivariate analysis of 416 patients with glioblastoma multiforme: prognosis, extent of resection, and survival. J Neurosurg 95(2):190-8 